\title{
LES COLLOCATIONS VERBO-NOMINALES ET LA TRADUCTION
}

\begin{abstract}
Vetulani Grażyna, Les collocations verbo-nominales et la traduction [Verb-noun collocations and translation]. Studia Romanica Posnaniensia, Adam Mickiewicz University Press, Poznań, vol. XXX: 2003, pp. 159-168. ISBN 83-232-1270-8. ISSN 0137-2475.

The main issue addressed in this paper is how to represent complex lexical structures in dictionaries. We focus on verb-noun type collocations. We argue in favour of the concept of lexicon-grammars as a useful, general framework to show how lexical units, simple or compound, behave within the language system. We postulate devclopment of lexicons grouping similar collocations in families according to a central meaning (common for each such family). Lexicons of that kind are precious not only as basic tools for human translators, but also as an essential part of all kinds of software contributing to the development of Human Language Technologies.
\end{abstract}

\section{INTRODUCTION}

Les travaux lexicographiques récents soulèvent souvent des problèmes liés à la fois à la forme des unités sémantiques de base et leur représentation dans un dictionnaire. Traditionnellement, les unités linguistiques (simples ou composées) sont insérées dans des citations littéraires (à la manière du Petit Robert) où elles apparaissent dans des segments plus ou moins courts, avec certains éléments cooccurrents. Aujourd'hui, la présentation du lexique se fait de plus en plus souvent sous forme de lexique-grammaire, c'est-à-dire sous forme de modèles rendant compte du fonctionnement des unités dans la langue. Ceci est conforme au fait que: «il n'est pas possible de donner le sens d'un mot sans utiliser une phrase, ni de contraster des emplois différents d'un même mot sans le placer dans des phrases» (Gross M. 1981: 48) ${ }^{1}$.

1 Pour le français, voir les travaux effectués au LADL à Paris 7, sous la direction de M. Gross et au LLI ì Paris 13, sous la direction de G. Gross. La bibliographie complète: Leclèrc C. (1998): Travaux récents en lexique-grammaire, Travaux de Linguistique, 37, Bruxelles, p. 155-186. 
Les remarques qui précèdent nous amènent à des réflexions sur des outils qui pourraient servir aux interprètes confrontés aux problèmes des choix lexicaux. Dans cet article, nous mettrons l'accent à la fois sur la construction des dictionnaires d'unités composées et les compétences des praticiens de traduction.

\section{BESOINS LIÉS A LA TRADUCTION}

Vu les activités commerciales et politiques d'aujourd'hui résultant en nombre croissant de documents traduits chaque jour, il n'est pas étonnant qu'on tâche de fournir aux traducteurs des outils spécialisés qui visent avant tout l'amélioration et l'accélération du processus de traduction ${ }^{2}$. Pour répondre à ces besoins, dans beaucoup de laboratoires, les chercheurs mettent l'accent sur l'importance de la confection des dictionnaires (parfois bien spécifiques) conçus en vue du traitement sur ordinateur. Ceci est imposé par les besoins liés à l'accès automatique aux données linguistiques. A l'heure actuelle, les possibilités techniques existent: les machines sont puissantes et il y a des corpus textuels stockés sous forme informatisée et étiquetée pour un traitement postérieur (Habert, Nazarenko, Salem 1997). En particulier, des efforts sont entrepris pour répondre aux besoins liés à la traduction assistée par ordinateur.

\section{DICTIONNAIRES MODERNES}

Pour créer des systèmes informatiques pouvant être utilisés dans la traduction, il est impératif de créer d'abord des dictionnaires recensant des unités linguistiques, conformément aux besoins du traitement automatique. Ce qui compte, c'est le type des unités dénombrées (toutes les unités doivent figurer dans un dictionnaire, aussi bien des mots simples que des composés, des expressions toutes faites, des collocations etc.) et la forme du codage. En général, on peut dire que les besoins de l'utilisateur imposent le type de l'information qui doit apparaître dans l'entrée du dictionnaire. Puisque les applications informatiques imposent qu'on fournisse toutes les informations nécessaires, même celles qui, pour l'homme, sont évidentes, la forme de la description est souvent spécifique. En tout cas, la description

${ }^{2}$ A titre d'illustration, notons seulement qu'à la Commission Européenne, la loi impose la traduction de plus de 3.000 .000 de pages par année. Soulignons aussi qu'il s'agit de 11 langues, et - en même temps - de beaucoup plus de combinaisons parce qu'on prend en considération des paires de langues correspondantes. 
faite pour les applications informatiques doit être beaucoup plus rigoureuse que celle que nous rencontrons dans les dictionnaires traditionnels où l'on peut compter sur les connaissances et l'intuition linguistiques du lecteur-homme ${ }^{3}$.

\section{UNITÉ COMPOSÉE EN TANT QU'UNE ENTRÉE DE DICTIONNAIRE}

Grâce aux banques de données linguistiques et aux outils informatiques existant, il est facile d'estimer aujourd'hui que dans une langue naturelle, il y a entre trois et quatre fois plus d'éléments composés que d'éléments simples (cf. Bogusławski 1976: 357, Gross G. 1989: 179). Cet état de choses ne se reflète pas pour autant dans les dictionnaires scolaires traditionnels, publiés sur papier, qui présentent presque exclusivement des mots simples. Un composé, constituant un signe linguistique, est de toute évidence une unité fondamentale. Alors, il doit constituer une entrée de dictionnaire, au même degré qu'une unité simple.

\subsection{COMPOSÉS JUXTAPOSÉS}

Dans la suite, nous nous concentrerons uniquement sur les composés juxtaposés (plus loin: juxtapositions ou tout simplement composés), laissant de côté des unités nommées fusions (en pol. zrost) et compositions (en pol. zlożenie) qui ont été l'objet d'une autre analyse (voir Vetulani G. 2000b).

Les juxtapositions (en pol. zestawienie) ne sont pas des groupes syntaxiques libres. Elles comportent deux (ou plusieurs) lexèmes concaténés formant ainsi une unité inséparable dans laquelle l'ordre des éléments est stable et dans laquelle chaque élément garde son accent et ses propriétés morphologiques. Bien que ce soient des éléments autonomes, leur recensement et description restent difficiles parce qu'on est confronté entre autres à des problèmes, comme:

a) la reconnaissance de l'unité de base (l'unité minimale): il faut reconnaître une telle unité dans un texte, c'est-à-dire vérifier si l'on a affaire à une unité sémantique (composée mais une seule unité) ou si c'est un groupe syntaxique libre; autrement dit, il s'agit de la reconnaissance du rattachement des constituants (selon l'analyse logique, cf. vase de Chine vs. vase /reçu/ de Chine); il est question de la bonne segmentation du texte en unités minimales,

b) la reconnaissance des règles de flexion à lintérieur du groupe; ceci est important dans les langues slaves qui sont hautement flexionnelles (par exemple en polonais le substantif a 3 genres, 2 nombres, 7 cas),

${ }^{3}$ Dans la littérature concernant la technologie du langage, on parle souvent des dictionnaires du type nouveau. Pour le français, voir entre autres: Courtois \& Silberztein (1988), Gross G. (1987), Guillet (199]), Silberztein (1993); pour le polonais, par exemple: Bogacki (1997), Vetulani G. (2000), Vetulani Z. (2000), Vetulani Z. \& Walczak B. \& Obrębski T. \& Vetulani G. (1998). 
c) la reconnaissance des possibilités de l'acceptation de certaines formes sur certaines positions (une unité composée peut comporter un mot qui, dans ce composé, fonctionne seulement sous une de toutes ses formes possibles).

Les juxtapositions comprennent:

a) des locutions figées parmi lesquelles on retrouve des unités terminologiques se rapportant à des objets, comme par exemple ziele angielskie (en pol.), pied noir (en fr.) ou des phrases entières (proverbes ou dictons), comme par exemple strach ma wielkie oczy (en pol.) ou faire feu de tout bois (en fr.),

b) des collocations, c'est-à-dire: «associations habituelles d'un morphème lexical avec d'autres au sein de l'énoncé, abstraction faite des relations grammaticales existant entre ces morphèmes: ainsi, les mots construction et construire, bien qu'appartenant à deux catégories grammaticales différentes, ont les mêmes collocations, c'est-à-dire qu'ils se rencontrent avec les mêmes mots» (Dubois et al. 1994: 91); parmi les collocations, on distingue des unités se rapportant à des objets ou états, comme par exemple blady strach, czarna rozpacz (en pol.), peur bleue (en fr.), mais aussi de très nombreuses structures verbo-nominales, comme par exemple pograżyć się $w$ lekturze, wysunać sugestie (en pol.) ou apporter un soutien, éprouver une difficulté (en fr.); les collocations, acceptant des variantes lexicales, se situent entre les structures figées et les groupes syntaxiques libres.

\subsubsection{COLLOCATION VERBO-NOMINALE EN' TANT QU'UNE ENTRÉE DE DICTIONNAIRE}

Dans les collocations verbo-nominales, les deux éléments constitutifs (c.-à-d. un verbe, suivi ou non d'une préposition et un nom abstrait, accompagné ou non d'un modifieur sous forme d'un adjectif ou d'un adverbe) constituent ensemble une expression prédicative. On pourrait y voir une sorte de 'verbe analytique ${ }^{4}$ ou, en tout cas, un tour prédicatif composé dans lequel le nom, étant un vrai prédicat sémantique, est supporté d'une sorte de verbe auxiliaire, nommé le plus souvent verbe support ou verbe de fonction (en allemand: Funktionsverben). Le nom est l'élément central de la phrase ce qui signifie qu'il accepte non seulement un certain verbe, mais qu'il sélectionne aussi des arguments au niveau de la phrase entière.

Nous avons affaire à un composé, une expression toute faite qui entre dans le système lexical de la langue pour des raisons conventionnelles et historiques ${ }^{5}$. Ceci dit, les éléments formant une telle expression sont souvent imprévisibles. Il est difficile d'expliquer par exemple pourquoi les noms tels que autorisation, baptême,

${ }^{4}$ C'est pourquoi dans la terminologie polonaise, ce groune de mots a reçu le nom de czasownik analityczny. Un autre nom c'est orzeczenie peryfrastyczne.

${ }^{5}$ C'est pourquoi, ce type d'expressions porte dans la littérature aussi le nom de syntagmes conventionnels (Szulc 1994: 109). 
démenti, acceptant tous les trois le verbe support donner, acceptent ensuite comme variantes stylistiques des formes diversifiées. Comparons: donner: une autorisation /le baptême / un démenti, mais: accorder une autorisation, administrer ou conférer le baptême et apporter un démenti.

Puisque les collocations verbo-nominales sont des unités distinctes et autonomes (chacune a son sens propre et est employée pour rendre des nuances sémantiques bien spécifiques), on pourrait les répertorier explicitement dans un dictionnaire spécialisé. La tâche s'avère pourtant difficile à cause:

a) du nombre très élevé de telles expressions dans une langue,

b) du fait que les unités analytiques soient peu représentées dans les dictionnaires traditionnels; si certains tours s'y trouvent, ijs sont disséminés dans le corps de l'article de l'un ou de l'autre constituant (Gross G. 1989: 179),

c) des cas de polysémie des noms prédicatifs,

d) des changements constants dans le lexique et des problèmes liés à l'acceptabilité des tours nouveaux, non standards, ceux qui sont à la mode et qui ne sont pas encore suffisamment lexicalisés pour être considérés comme normatifs ou conscillés pour l'usage scolaire par exemple,

e) des possibilités du recensement de telles unités; il y a des difficultés aussi bien au niveau de l'accès aux corpus représentatifs (pour certaines langues, les corpus nationaux n'existent pas) qu'au niveau des outils de recherches performants.

Malgré tous ces problèmes, avec un succès plus ou moins grand pour différentes langues, on construit des dictionnaires spécifiques sous forme de tables sémantico-syntaxiques où l'on introduit l'unité en question avec, en même temps, l'information grammaticale sur son emploi. Dans la pratique, il est commode de décrire en premier lieu les prédicats (verbaux, nominaux, adjectivaux, adverbiaux) parce qu'ils se laissent définir grâce aux éléments qu'ils sélectionnent dans la structure d'emploi, notamment dans une phrase simple ${ }^{6}$. L'étude des noms prédicatifs permet d'observer en premier lieu les verbes cooccurrents. L'analyse systématique des substantifs du polonais (Vetulani G. 2000a) a permis d'extraire un sousensemble d'environ 8000 noms prédicatifs (tous abstraits), divisés ensuite en 5 classes de taille et d'homogénéité variées ${ }^{7}$. Bien que les prédicats nominaux se laissent en général classifier en fonction du verbe support, dans le cas de la langue polonaise, la classification précise et détaillée pose beaucoup de problèmes car:

${ }^{6}$ La méthodologie lexique-grammaire a ćté appliquéc avec succès pour le français (tous les prédicats sont recensés ct décrits), mais le problème n'est pas spécifique au français ni aux langues romanes, ni même aux langues indo-européennes (des travaux ont été effcctués par cxcmple pour le japonais, le coréen et beaucoup d'autres langues).

${ }^{7}$ La Classe I contient les noms désignant tout type d'activité et de comportement (opérations, techniques, méthodes, états, processus), la Classe If: les noms se rapportant aux traits de de caractère, la Classe III: les noms désignant les maladies, la Classe IV: les noms désignant les professions, la Classe V: les noms qui entrent dans les constructions avec un verbe support événementicl. 
a) les verbes accompagnant les noms abstraits sont très nombreux (pratiquement tous pour un de leurs emplois) ${ }^{8}$,

b) ils sont souvent très recherchés stylistiquement (une classification n'est pas pourtant impossible, mais les classes seraient beaucoup plus multiples qu'en français où les noms se regroupent naturellement autour des verbes neutres, tels que: faire, avoir, être en, donner, recevoir etc.),

c) les verbes, par le jeu des préfixes et suffixes, fournissent l'information concernant l'aspect grammatical et le mode d'action; toutes les formes possibles ne sont pas acceptées par un nom donné; un nom prédicatif peut imposer au verbe une catégorie grammaticale déterminée, comme par exemple un aspect seulement de la paire aspectuelle: perfectif / imperfectif (cf. przywiazywac wage ou toczyć dyskusje et *przywiazać wage, *stoczyć dyskusję).

Les collocations verbo-nominales pourraient être dénombrées pour les besoins pratiques. On arriverait ainsi à résoudre les problèmes liés à la polysémie des noms entrant dans ces groupes. Analysons par exemple le nom komenda qui, en fonction du verbe cooccurrent, peut avoir le sens de 'ordre' ou 'commandement':

1. wydać komende (do ataku) (donner l'ordre de...)

2. abjać komendę (nad wojskiem) (avoir le commandement de..., prendre la direction de...)

Nous retrouvons la même situation en allemand:

1. ein Kommando geben

2. das Kommando führen (über)

La présentation du nom prédicatif dans des segments du type verbe support + nom abstrait, comme celle que nous venons d'observer peut s'avérer pourtant insuffisante. Il arrive notamment qu'une expression prédicative entière (verbe + nom), sans contexte, reste encore ambiguë. Nous pouvons observer ce phénomène par exemple en polonais dans l'expression mieć chody qui peut recevoir le sens de 'marcher vite' (cf. Ależ on ma chody! Ani go dogonić!) ou 'être pistonné' (cf. Jan ma chody $w$ ministerstwie, Jan ma chody $u$ dyrektora). Il en résulte que seul le recours à la structure entière (une phrase complète) peut rendre compte des sens différents d'une unité lexicale. Dans le cas des noms prédicatifs, pour identifier les sens différents, il ne suffit pas d'observer les verbes cooccurrents, mais il faut prendre en considération aussi d'autres éléments importants, tels que le nombre d'arguments sélectionnés, leurs traits grammaticaux (la nature du sujet et des compléments), la façon dont ils apparaissent dans la phrase (avec ou sans préposition) ou d'autres éléments encore, comme par exemple la détermination du nom prédicatif. Comparons à titre d'illustration:

\footnotetext{
${ }^{8}$ Voir aussi Jędrzejko (1998: 50).
} 

1. fém. sg.; *fém. pl.:
Luc a raison de parler
2. fém.sg.; fém. pl.:
Luc a une raison de parler
Luc a des raisons de parler
3. *fém. sg.; fém. pl.:
Luc a ses raisons de parler

\subsubsection{PROUUCTIVITÉ DES NOMS PRÉDICATIYS}

Un nom prédicatif admet souvent plusieurs verbes. Les choix s'effectuent en fonction des sens différents. A côté des emplois où le verbe est un indicateur du sens nouveau du prédicat nominal, nous pouvons observer des cas où il modifie seulement le sens de base. Autrement dit, pour un sens de base donné, un nom prédicatif accepte souvent un grand nombre de variantes. Ce sont des variantes d'ordre stylistique (variantes neutres, recherchées, vulgaires), temporel ou aspectuel. Les groupes verbo-nominaux, acceptant des échanges à la position du verbe, sont donc des groupes très productifs. Comparons les familles autour de deux noms en polonais, notamment apatia (apathie) et tempo (allure):

apatia: $\quad$ być $w$ stanie apatii / bywać w stanie apatii

popaść / popadać $w$ apatię

ulec / ulegać apatii

poddać się / poddawać się apatii

pogrązyć się / pogrązać się w apatii

tempo: mieć tempo (+ un modifieur)

brać / wziqć tempo (+ un modifieur)

trzymać tempo

utrzymać / utrzymywać

Dans chaque famille, le sens fondamental reste le même pour toutes les expressions. Ainsi les expressions partagent un élément sémantique, mais diffèrent par les sens additionnels apportés par les verbes (Lewicki, 1996: 13). Pour nuancer le sens, un usager de la langue recourt soit à une forme lexicale nouvelle (un autre verbe), soit à un procédé dérivationnel (un préfixe ou un suffixe) selon le caractère de la forme verbale ${ }^{9}$. Chaque tour constitue une collocation nouvelle. Il serait utile de répertorier dans un dictionnaire toutes les collocations possibles créées autour d'une forme nominale.

Analysons encore de ce point de vue une autre forme en polonais, notamment le prédicat nominal mandat qu'on peut présenter dans deux familles différentes en fonction des sens de base différents:

9 Dans les familles présentées, nous avons mis dans la même ligne les formes exprimant les aspects grammaticaux: perfectif/imperfectif. Les variantes apparaissant dans les lignes consécutives sont des variantes stylistiques. 
1. au sens de 'donner un certain mandat à qq.':

dać mandat (komuś, jakiś)

udzielić mandatu (komuś, jakiegoś) - plus recherché

2. au sens de 'procès-verbal': donner une amende à qq., pour...

dać mandat komuś za coś

wymierzyć mandat komuś za coś - plus recherché: infliger... wlepić mandat komuś za coś - familier: coller...

Nous observons le même phénomène en allemand. Prenons par exemple das Kommando:

1. au sens de 'direction':

das Kommando führen (über)

das Kommando haben (über)

das Kommando übernehmen

2. au sens de 'ordre':

ein Kommando geben

La façon de présenter sous forme d'un inventaire complet des structures verbonominales permet de rendre compte en même temps de la richesse du système lexical dans le domaine des tours analytiques et de la productivité de ce type de constructions. En plus, on peut aussi rendre compte d'une manière pratique des sens totalement différents (autant de sens de base différents que de familles différentes). Pour décrire les possibilités lexicales dans ce domaine, il nous paraît utile de faire appel à ce que Lewicki [ibidem] appelle grille dérivationnelle (cf. Vetulani, à paraître $)^{10}$. Enfin, le recensement exhaustif seul peut démontrer ce qui est acceptable dans une langue et quels sens différents peut avoir une forme donnée.

A la base des dictionnaires recensant des unités de la manière présentéc, on peut concevoir des logiciels qui présenteraient aux traducteurs des listes de propositions concrètes. D'une liste, le traducteur choisirait une expression, celle qui lui conviendrait le mieux pour le texte à traduire. La bonne reconnaissance et l'utilisation active correcte des collocations sont le signe de la compétence linguistique et traductologique en langue étrangère. En constituant des listes de collocations, on donnerait à la disposition des interprètes l'inventaire des tours en même temps selon les registres de la langue et les catégories grammaticales acceptables. Comme les collocations sont pratiquement imprévisibles, les interprètes choisiraient du moins des expressions correctes. La confection de dictionnaires de ce type, ainsi que des outils informatiques dérivés de ces dictionnaires, pourrait alors contribuer à la fois à l'amélioration et l'accélération du processus de traduction.

\footnotetext{
${ }^{10}$ Le terme polonais: siatka derywacyjna.
} 


\section{CONCLUSION}

Un bon interprète doit tenir compte des emplois normatifs et courants, des collocations figées et de celles qui sont en train de se lexicaliser, des expressions dans l'usage commun et de celles qui sont employées seulement dans certains registres ou par certains groupes sociaux, de celles enfin qui ne sont pas encore généralement acceptées. Aux difficultés liées au choix d'une expression complexe parmi d'autres expressions complexes s'ajoutent des problèmes concernant l'équivalence entre les tours analytiques et leurs correspondants synthétiques. On sait par exemple qu'en polonais, les tours analytiques sont plus fréquents dans le langage scientifique et journalistique (dans les styles qui visent une précision et une représentation mentale), tandis qu'en français, comme l'ont montré les travaux effectués au LADL, ils sont avant tout d'usage neutre (cf. udzielić rady et donner un conseil). Bien que la langue polonaise soit très riche en collocations verbonominales, on y trouve souvent un verbe à l'endroit où le français emploie un tour analytique (cf. plażować et faire de la plage, przejść się et faire un tour) ${ }^{11}$.

\section{BIBLIOGRAPHIE}

Bogacki. K. (1997), POLLEX - un dictionnaire électronique morphologique dis polonais, BULAG, Grenoble.

Boguslawski, A. (1996), O zasadach rejestracji jednostek jezzyka, Poradnik Językowy, 8, pp. 356364.

Courtois, B. \& Silberztein, M. (1989), Les dictionnnires électroniques DELAS et DELAC, Actes du colloque sur les langues romanes, Université LAVAL, Québec, pp. 69-94.

Dubois, J. (ćd.) (1994), Dictionnaire de linguistique et des sciences du langage, Laroussc, Paris.

Gross, G. (1989), Le dictionnaire et l'enseignement de la langue maternelle, Wörterbücher Dictionarics Dictionnaircs, Walter de Gruyter, Berlin-New York;

Gross, G. \& Vivès, R. (ćd.) (1986), Syntaxe des noms. Languc Française, 69.

Gross, M. (1981), Les bases empiriques de la notion de prédicat sémantique, Langages, 63:7-52;

Guillet, A. (1991), Dictionnaires électroniques et lexique-grammaire, Studia Romanica Posnaniensia, XVI, UAM, Poznań, pp. 117-128.

Habert, B. \& Nazarenko, A. \& Salem, A. (1997), Les linguistiques de corpus, Armand Colin, Paris.

Jędrzejko, E. (1998), Slownik polskich zwrotów werbo-nominalnych. Zeszyt próbny, Energeia, Warszawa.

Lewicki, A. M. (1976), Wprowadzenie do frazeologii syntaktycznej. Teoria zwrotu frazeologicznego, Katowice.

Lewicki, A.M. (1977), Zwroty frazeologiczne, czyli predykaty w formie skladników nieciaglych, Studia Gramatyczne, I: 135-143.

${ }^{11}$ Ce type de problèmes n'apparaît pas évidemment au moment où la langue cible dispose seulement d'un genre d'expressions là où la langue source en a plusieurs (cf. en français: espérer el avoir de l'espoir, par contre en polonais: mieć nadzieje sculcment). 
Lewicki , A.M. (1982) (éd.), Stalość i zmienność zwiqzków frazeologicznych, Lublin.

Lewicki, A.M. (1996), Relacyjna siatka derywacyjna jako czynnik onomazjologicznego opisu frazeologicznego, Problemy frazeologii europejskiej, I, Energeia, Warszawa, s. 9-14.

Silberztein, M. (1993), Dictionnaires électroniques et analyse automatique de textes. Le système INTEXT, MASSON, Paris, Milan, Barcelone, Bonn.

Vetulani, G. (2000a), Rzeczowniki predykatywne języka polskiego. W kierunku syntaktycznego slownika rzeczowników predykatywnych. Wydawnictwo Naukowe UAM, Poznań.

Vetulani, G. (2000b), Sur un certain type de composés, K. Dorfmüller-Karpusa \& E. Vretta-Panidou (eds.), Thessaloniker Inerkulturclle Analysen, Akten des 33. Linguistischen Kolloquiums in Thessaloniki 1998, Peter Lang, pp. 375-382.

Vetulani, G. $(2000 \mathrm{c})$, Quelques exemples d'analyse des corpus en vue de la traduction, Studia Romanica Posnaniensia, XXV/XXV1, UAM, Poznań, pp. 317-325.

Vetulani, G. (à paraitrc), Collocations verbo-nominales: structure d'une entrée de dictionnaire, Akten des 36. Linguistischen Kolloquiums in Ljubljana 2001, Pctcr Lang.

Vetulani, Z. (2000), Electronic Language Resources for Polish: POLEX, CEGLEX, GRAMLEX, $M$. Gavrilidou et al. (eds.), Proceedings of Second International Conference on Language Resources and Evaluation, Athens, Greece, ELRA, pp. 367-374.

Vetulani, Z. \& Walczak, B. \& Obrębski, T. \& Vetulani, G. (1998), Unambiguous coding of the inflection of Polish nouns and its application in electronic dictionaries - forma POLEX. Jednoznaczne kodowanie fleksji rzeczownika polskiego $i$ jego zastosowanie $w$ slownikach elektronicznych-format POLEX, UAM, Poznań. 